\title{
Gene Effects for Yield and Yield Components in Chickpea (Cicer arietinum L.) under Irrigated and Rainfed Conditions
}

\author{
B. L. Kumhar ${ }^{1}$, D. Singh ${ }^{2}$, T. B. Bhanushally ${ }^{1} \&$ N. R. Koli ${ }^{3}$ \\ ${ }^{1}$ Agricultural Research Sub Station, Hanumangarh Town, India \\ ${ }^{2}$ Department of Plant Breeding and Genetics, SKN College of Agriculture, Jobner, India \\ ${ }^{3}$ Agricultural Research Station, Kota, India \\ Correspondence: B. L. Kumhar, College of Agriculture, Lalsot (Dausa)-303 511, Rajasthan, India. E-mail: \\ kumharb171@gmail.com
}

Received: August 10, 2012 Accepted: October 8, 2012 Online Published: February 5, 2013

doi:10.5539/jas.v5n3p1 URL: http://dx.doi.org/10.5539/ jas.v5n3p1

\begin{abstract}
Seven genetically diverse parents of chickpea were crossed in five combinations (RSG-895 x RSG-888, RSG-888 x ICC-4958, IPC-94-94 x RSG-888, CSJD-901 x RSG-931 and BG-362 x RSG-931) to develop $F_{1}$, $F_{2}$ and $\mathrm{F}_{3}$ hybrid progenies. Five generations viz., $\mathrm{P}_{1}, \mathrm{P}_{2}, \mathrm{~F}_{1}, \mathrm{~F}_{2}$ and $\mathrm{F}_{3}$ were grown in a compact family block design under irrigated and rainfed conditions to estimate the gene effects for yield and yield components. Scaling test ' $\mathrm{C}$ ' and ' $\mathrm{D}$ ' as well as joint scaling test revealed presence of epistatic in all the crosses for all the characters studied viz., days to $50 \%$ flowering, days to maturity, plant height, fruiting branches per plant, pods per plant, seeds per pod, biological yield per plant, seed yield per plant, harvest index, 100-seed weight and protein content under both the conditions. Both main effects additive (d) and dominance (h) were important for all the characters in all the crosses under both the conditions except 100-seed weight, where only additive effect was found important. Both additive $\mathrm{x}$ additive (i) and dominance $\mathrm{x}$ dominance (1) interaction effects were also important for all the characters in all the crosses under both the conditions except 100-seed weight, where only additive $\mathrm{x}$ additive interaction was found important. Generally, the magnitudes of dominance (h) and dominance x dominance (l) were prevailed over additive (d) and additive $\mathrm{x}$ additive (i) effects, respectively. Duplicate type of epistasis was observed in all the cases, where epistasis was established. Thus, it can be concluded that additive, dominance, additive $\mathrm{x}$ additive (i) and dominance $\mathrm{x}$ dominance (1) effects contributed significantly to the inheritance of various component characters in chickpea under both irrigated and rainfed conditions. These results implies the use of recurrent selection by way of intermating the desirable segregants followed by selection or biparental approach/intermating of segregants in early segregating generations for improvement of these components characters in chickpea under both the conditions.
\end{abstract}

Keywords: chickpea, gene effects, epistasis, inheritance, Cicer arietinum

\section{Introduction}

Chickpea (Cicer arietinum L.) is the third most important pulse crop (after dry bean and pea) and currently grown on about 10 mha world wide, with $95 \%$ cultivation in the developing countries. In Asia, India accounts for $65.3 \%$ of the area and $67.2 \%$ of the production. Chickpea has special significance in the diet of the predominantly vegetarian population of India as it contains more protein, which is complementary with cereals in amino acids profile. However, production and productivity of chickpea have been stagnant for the past three decades. One of the main reason is its sensitive to moisture stress at critical stages as more than $80 \%$ area under chickpea is rainfed.

The precise knowledge of the nature of gene action for yield attributing traits helps in the choice of an effective breeding strategy to accelerate the pace of genetic improvement of seed yield. Most of the reports for gene action in chickpea are based on the diallel mating (Katiyar \& Singh, 1980, Deshmukh \& Bhapkar, 1982) which does not provide information regarding non-allelic gene actions. The non-allelic gene actions could inflate the measures of additive and dominance components. Further, Toledo et al. (1991) suggested that the fiveparameter model was good as the back cross studies for estimation of gene effects and gives satisfactory results. 
Keeping this in mind, the present investigation was carried out to determine the gene effects for yield and yield components in five crosses of chickpea under irrigated and rainfed conditions through generation mean analysis.

\section{Materials and Methods}

\subsection{Plant Material}

Seven desi chickpea cultivars viz., RSG-895, RSG-888, ICC-4958, IPC-94-94, CSJD-901, RSG-931 and BG-362 of diverse pedigree, seed size origin and agro-climatic adaptation were crossed in five combinations viz., RSG-895 x RSG-888, RSG-888 x ICC-4958, IPC-94-94 x RSG-888, CSJD-901 x RSG-931 and BG-362 x RSG-931. Five generations viz., $\mathrm{P}_{1}, \mathrm{P}_{2}, \mathrm{~F}_{1}, \mathrm{~F}_{2}$ and $\mathrm{F}_{3}$ of these five crosses were grown in a compact family block design with three replications during rabi 2004-05 under both irrigated (two supplemental irrigations) and rainfed (on receding soil moisture i.e. conserved moisture from rainfall prior sowing which was $122.6 \mathrm{~mm}$ ) at Research Farm, Agricultural Research Sub Station, Hanumangarh. Seeds were sown in 3 meter long rows. Keeping spacing between and within rows at $0.30 \mathrm{~m} \times 0.10 \mathrm{~m}$. Parents were represented by two rows, $\mathrm{F}_{1} \mathrm{~s}$ by one row, $\mathrm{F}_{2} \mathrm{~S}$ and $\mathrm{F}_{3} \mathrm{~s}$ by four rows. Observations for eleven yield related characters viz., days to $50 \%$ flowering, days to maturity, plant height, fruiting branches per plant, pods per plant, seeds per pod, biological yield per plant, seed yield per plant, harvest index, 100-seed weight and protein content were recorded on 10 randomly selected plants in $\mathrm{P}_{1}, \mathrm{P}_{2}$ and $\mathrm{F}_{1}$ generations and on 20 plants in $\mathrm{F}_{2}$ and $\mathrm{F}_{3}$ generations.

\subsection{Statistical Analysis}

ANOVA was performed as per compact family block design for comparison of crosses as well as generations of each cross. Pooled analysis of variance was also done over two environments (Irrigated and rainfed) according to Panse and Sukhatme (1985). The scale tests 'C' and ' $D$ ' (Mather, 1949) were applied to test the presence or absence of non-allelic interaction. Joint Scaling (Cavalli, 1952) was applied to find out the presence of interaction and estimate the $\mathrm{m},(\mathrm{d})$ and (h) parameters. Significant $\chi^{2}$ - values of joint scaling test suggested the inadequacy of additive-dominance model and it was considered appropriate to use five-parameter model of Hayman (1958) for the estimation of gene effects under both the conditions.

\section{Results and Discussion}

The mean squares from ANOVA presented in Table 1 showed that there were significant differences among crosses as well as in generations. The pooled analysis of variance over environments (irrigated and rainfed) presented in Table 2, revealed highly significant differences between environments for all the characters in all the crosses except for seeds per pod in cross RSG-888 x ICC-4958 and BG-362 x RSG-931. Significant differences between the environments, indicating the effect of environments on expression of characters. Generation $\mathrm{x}$ Environment interaction also significant for most of the characters in all the crosses except for seeds per pod in RSG-895 x RSG-888, RSG-888 x ICC-4958 and BG-362 x RSG-931, for 100-seed weight in RSG-888 x ICC-4958, IPC-94-94 x RSG-888 and BG-362 x RSG-931 and protein content in RSG-895 x RSG-888 and RSG-888 x ICC-4958, which indicated the variable effect of environments on the expression of characters. The scaling tests ' $C$ ' and ' $D$ ' and Joint Scaling test ( $\chi^{2}$ - values) indicated the presence of epistatic interactions in all the crosses for all the characters under both the conditions (Table 3 ). The genetic architecture of individual characters is discussed below.

\subsection{Days to $50 \%$ Flowering}

Both additive (d) and dominance (h) effects were significant in all the crosses under both the conditions except for dominance in IPC-94-94 x RSG-888 under rainfed, with a greater magnitude of dominance than additive, indicating predominance of dominance for days to $50 \%$ flowering. Similarly, both additive $\mathrm{x}$ additive (i) and dominance $\mathrm{x}$ dominance (1) interactions were significant in all the crosses under both the conditions except for additive $\mathrm{x}$ additive in RSG-895 x RSG-888 and CSJD-901 x RSG-931 and dominance $\mathrm{x}$ dominance in IPC-94-94 x RSG-888 in irrigated condition. Higher magnitude of dominance $x$ dominance than additive $x$ additive indicated the importance of dominance $x$ dominance (1) for inheritance of this trait. 
Table 1. Analysis of variance (mean squares) for different characters in five generations and five crosses of chickpea under irrigated (IRG) and rainfed (RF) conditions as per compact family block design

Between crosses:

\begin{tabular}{|c|c|c|c|c|c|c|}
\hline \multirow{2}{*}{ Characters } & \multicolumn{3}{|l|}{ IRG } & \multicolumn{3}{|l|}{$\mathrm{RF}$} \\
\hline & Rep. (2 df) & Crosses (4 df) & Error (8 df) & Rep. (2 df) & Crosses (4 df) & Error (8 df) \\
\hline $\begin{array}{l}\text { Days to } 50 \% \\
\text { flowering }\end{array}$ & 0.070 & $51.708^{* *}$ & 0.096 & 0.037 & $198.05 * *$ & 0.081 \\
\hline Days to maturity & 0.174 & $88.793^{* *}$ & 0.316 & 0.167 & $147.186^{* *}$ & 0.033 \\
\hline Plant height $(\mathrm{cm})$ & 0.058 & $55.084 * *$ & 0.449 & 0.148 & $19.633^{* *}$ & 0.231 \\
\hline $\begin{array}{l}\text { Fruiting branches } \\
\text { per plant }\end{array}$ & 0.258 & $1.455^{* *}$ & 0.172 & 0.043 & $6.927 * *$ & 0.106 \\
\hline Pods per plant & 0.578 & $24.465^{* *}$ & 1.405 & 1.281 & $52.503^{* *}$ & 0.499 \\
\hline Seeds per pod & 0.002 & $0.045^{* *}$ & 0.001 & 0.003 & $0.019 * *$ & 0.001 \\
\hline $\begin{array}{l}\text { Biological yield per } \\
\text { plant }(\mathrm{g})\end{array}$ & 0.365 & $19.267 * *$ & 0.318 & 0.478 & $22.987 * *$ & 0.271 \\
\hline $\begin{array}{l}\text { Seed yield per plant } \\
(\mathrm{g})\end{array}$ & 0.131 & $7.788^{* *}$ & 0.130 & 0.089 & $18.029 * *$ & 0.219 \\
\hline Harvest index $(\%)$ & 0.248 & $7.848 * *$ & 0.603 & 0.251 & $51.117 * *$ & 0.205 \\
\hline 100 -seed weight $(\mathrm{g})$ & 0.098 & $35.574 * *$ & 0.086 & 0.030 & $29.679 * *$ & 0.165 \\
\hline Protein content $(\%)$ & 0.016 & $0.622 * *$ & 0.023 & 0.005 & $0.689 * *$ & 0.008 \\
\hline
\end{tabular}

Between generations within crosses:

\begin{tabular}{|c|c|c|c|c|c|c|}
\hline \multirow{2}{*}{ Characters } & \multicolumn{3}{|l|}{ IRG } & \multicolumn{3}{|l|}{$\mathrm{RF}$} \\
\hline & Rep. (2 df) & Gener. (4 df) & Error (8 df) & Rep. (2 df) & Gener. (4 df) & Error ( $8 \mathrm{df})$ \\
\hline
\end{tabular}

Days to $\mathbf{5 0 \%}$
flowering

RSG-895 $\quad \mathrm{x}$

RSG-888

$\mathrm{X}$

RSG-888

ICC-4958

$\mathrm{X}$

IPC-94-94

$\mathrm{x}$

RSG-888

CSJD-901 $\quad x$

RSG-931

BG-362 x RSG-931

$$
1.445
$$

$18.681 * *$

2.090

0.002

$7.390 * *$

0.916

Days to maturity

$\begin{array}{lr}\text { RSG-895 } & x \\ \text { RSG-888 } & \\ \text { RSG-888 } & x \\ \text { ICC-4958 } & \\ \text { IPC-94-94 } & x \\ \text { RSG-888 } & \\ \text { CSJD-901 } & \text { x } \\ \text { RSG-931 } & \end{array}$

BG-362 x RSG-931

$$
0.048
$$

$12.398 * *$

1.176

0.269

$12.164 * *$

1.016

$0.064 \quad 319.744 * * \quad 3.233$

0.868

$242.273^{* *}$

3.534

0.452

$4.144^{*}$

0.850

0.200

$7.599 * *$

0.783

0.266

$8.126^{* *}$

0.850

0.464

$22.468 * *$

0.633

\section{$\mathrm{x}$}

1364

$11.592 *$

1.947

0.171

$16.669 * *$

0.640

2.561

$24.823 * *$

0.779

0.061

$17.013 * *$

0.907

2.399

$250.044 * *$

13.900

0.198

$277.193 * *$

3.117

Plant height (cm)

RSG-895 
RSG-888

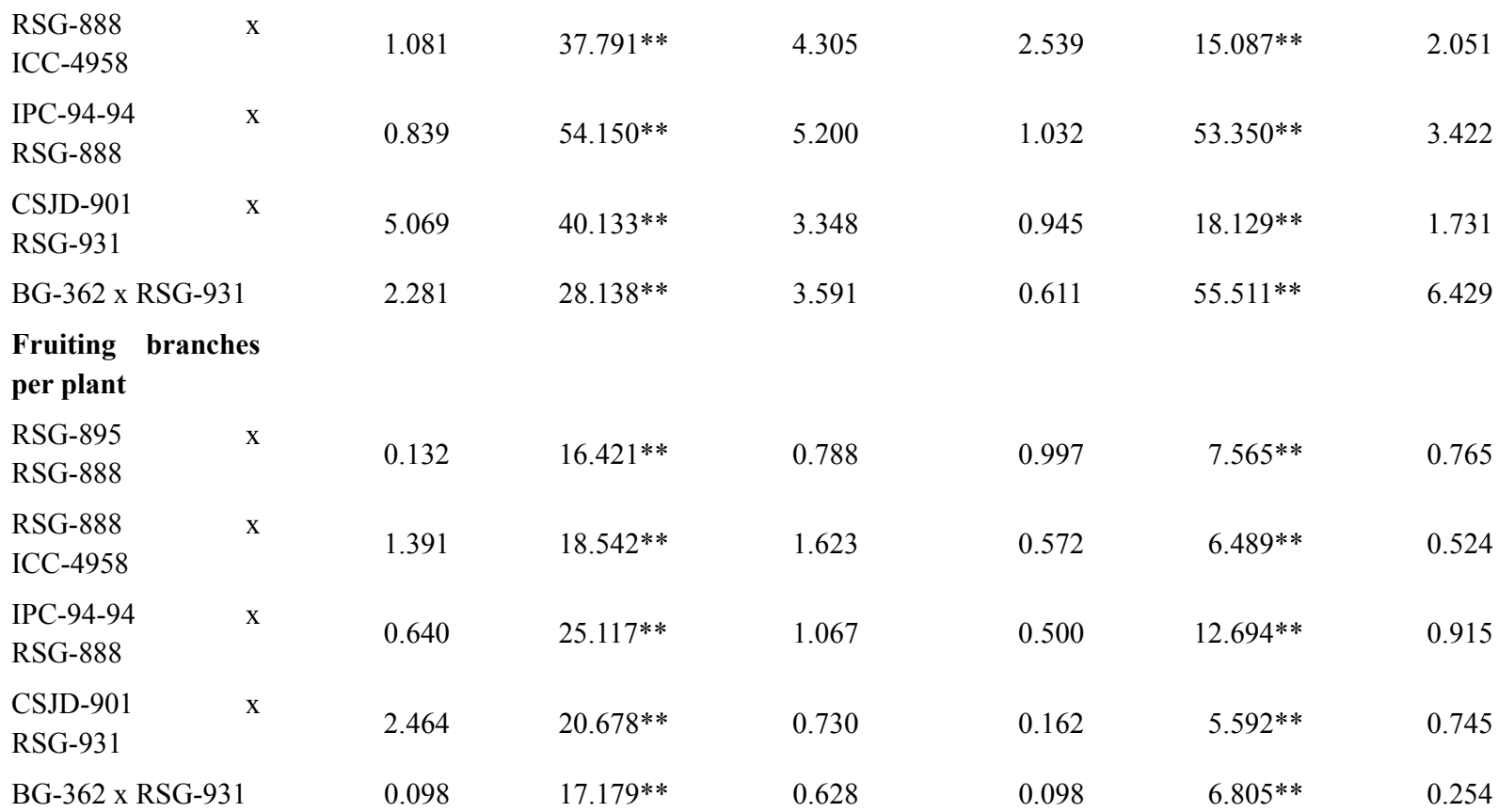

\section{Pods per plant}

RSG-895

RSG-888

13.089

$331.556^{* *}$

16.37

1.237

$173.984 * *$

3.208

RSG-888 $\quad \mathrm{x}$

ICC-4958

1.798

$142.749 * *$

12.227

4.862

$263.506^{* *}$

3.416

IPC-94-94 $\quad x$

RSG-888

3.174

$151.826^{* *}$

9.067

1.335

$152.074 * *$

6.353

CSJD-901 $\mathrm{x}$

RSG-931

9.204

$115.315^{* *}$

14.863

7.074

$177.750 * *$

6.860

BG-362 x RSG-931

3.725

$127.541 * *$

10.562

1.866

$147.495^{* *}$

7.105

Seeds per pod

$\begin{array}{ll}\text { RSG-895 } & \text { x } \\ \text { RSG-888 } & \\ \text { RSG-888 } & \text { x } \\ \text { ICC-4958 } & \\ \text { IPC-94-94 } & \\ \text { RSG-888 } & \\ \text { CSJD-901 } \\ \text { RSG-931 }\end{array}$

BG-362 x RSG-931

0.016

$0.041^{*}$

0.008

0.006

$0.034 * *$

0.004

0.002

$0.078^{* *}$

0.011

0.007

$0.076^{* *}$

0.004

$0.043 *$

0.008

0.003

$0.045 * *$

0.001

$0.011 \quad 0.064 * *$

0.009

0.001

$0.019 * *$

0.001

0.003

$0.024 * *$

0.003

0.003

$0.039^{* *}$

0.003

Biological yield per plant (g)

RSG-895

RSG-888

2.423

$45.621 * *$

2.995

1.991

$31.965 * *$

2.133

RSG-888

0.992

$68.477^{* *}$

2.640

0.879

$81.273 * *$

1.912

IPC-94-94 $\mathrm{x}$

RSG-888

0.084

$50.796^{* *}$

2.072

1.487

$33.537^{* *}$

2.784 


$\begin{array}{lrrrrrr}\begin{array}{l}\text { CSJD-901 } \\ \text { RSG-931 }\end{array} & 3.787 & 28.793 * & 4.187 & 0.917 & 30.259 * * & 1.191 \\ \text { BG-362 x RSG-931 } & 0.904 & 76.659 * * & 4.451 & 2.529 & 38.431 * * & 2.148\end{array}$

\section{Seed yield per plant}

(g)

RSG-895

RSG-888

0.588

$20.359 * *$

1.200

1.666

$15.436^{* *}$

0.738

RSG-888 x

ICC-4958

0.117

$27.273 * *$

1.123

1.336

$41.168 * *$

0.885

IPC-94-94 $\mathrm{x}$

RSG-888

0.722

$12.402 * *$

1.194

0.578

$19.553 * *$

0.762

CSJD-901 $\mathrm{x}$

RSG-931

1.274

$10.832 * *$

0.867

0.989

$6.320 * *$

0.345

BG-362 x RSG-931

0.566

$25.112 * *$

1.242

0.259

$30.401 * *$

1.415

Harvest index (\%)

$\begin{array}{lr}\text { RSG-895 } & \text { x } \\ \text { RSG-888 } & \\ \text { RSG-888 } & \text { x } \\ \text { ICC-4958 } & \\ \text { IPC-94-94 } & x \\ \text { RSG-888 } & \\ \text { CSJD-901 } & \text { x } \\ \text { RSG-931 } & \end{array}$

BG-362 x RSG-931

$\mathrm{x}$

10.479

$67.467^{* *}$

5.798

1.283

$66.859 * *$

3.276

1.297

$77.514 * *$

1.359

1.807

$71.264 * *$

1.842

0.629

$28.351^{* *}$

1.514

0.591

$62.157 * *$

1.106

$0.200 \quad 16.665^{*}$

2.504

0.258

$13.102 * *$

1.384

100-seed weight (g)

$\begin{array}{lr}\text { RSG-895 } & \text { x } \\ \text { RSG-888 } & \\ \text { RSG-888 } & \mathrm{x} \\ \text { ICC-4958 } & \\ \text { IPC-94-94 } & \mathrm{x} \\ \text { RSG-888 } & \\ \text { CSJD-901 } & \mathrm{x} \\ \text { RSG-931 } & \end{array}$

0.682

$37.732 * *$

1.614

1.428

$91.035 * *$

1.379

$\mathrm{X}$

0.061

$2.157 * *$

0.094

0.326

$1.972 * *$

0.060

$\mathrm{x}$

1.256

$75.130 * *$

4.095

0.083

$57.462 * *$

0.503

0.310

$39.523 * *$

2.176

1.515

$28.635^{* *}$

1.011

RSG-931

0.127

$2.588^{* *}$

0.144

0.163

$3.884 *$

0.643

BG-362 x RSG-931

0.461

$44.749^{* *}$

0.939

1.362

$34.737 * *$

0.654

Protein content

(\%)

RSG-895 $\mathrm{x}$

RSG-888

0.183

$1.145^{*}$

0.202

0.064

$0.946^{* *}$

0.066

RSG-888 $\quad x$

ICC-4958

0.055

$1.929 * *$

0.049

0.018

$1.258^{* *}$

0.041

IPC-94-94 X

RSG-888

0.059

$4.952 * *$

0.094

0.055

$3.812 * *$

0.061

CSJD-901 $\mathrm{x}$

RSG-931

0.193

$2.515^{* *}$

0.192

0.033

$0.917 * *$

0.027

BG-362 x RSG-931

0.052

$1.659 * *$

0.191

0.019

$0.132 * *$

0.017

*, ** significant at 5 per cent and 1 per cent level, respectively 
Table 2. Pooled analysis of variance (mean squares) in five chickpea crosses over two environments (irrigated and rainfed)

\begin{tabular}{|c|c|c|c|c|c|}
\hline Characters/crosses & $\begin{array}{l}\text { Env.(E) } \\
\text { (1df) }\end{array}$ & $\begin{array}{l}\text { Rep./ Env. } \\
(4 \mathrm{df})\end{array}$ & $\begin{array}{l}\text { Gener.(G) } \\
(4 \mathrm{df})\end{array}$ & $\begin{array}{l}\text { Gx E } \\
(4 \mathrm{df})\end{array}$ & $\begin{array}{l}\text { Error } \\
(16 \mathrm{df})\end{array}$ \\
\hline \multicolumn{6}{|l|}{ Days to $50 \%$ flowering } \\
\hline RSG-895 x RSG-888 & $412.799 * *$ & 0.722 & $9.586^{* *}$ & $16.483 * *$ & 1.504 \\
\hline RSG-888 x ICC-4958 & $208.086^{* *}$ & 0.159 & $20.624 * *$ & $3.938^{*}$ & 1.096 \\
\hline IPC-94-94 x RSG-888 & $1320.254 * *$ & 0.466 & $428.814 * *$ & $133.202 * *$ & 3.384 \\
\hline CSJD-901 x RSG-931 & $265.083 * *$ & 0.326 & $7.858 * *$ & $3.885^{*}$ & 0.816 \\
\hline BG-362 x RSG-931 & $40.756^{* *}$ & 0.366 & $26.680 * *$ & $3.913 * *$ & 0.741 \\
\hline \multicolumn{6}{|l|}{ Days to maturity } \\
\hline RSG-895 x RSG-888 & $418.133 * *$ & 0.766 & $20.391 * *$ & $7.868 * *$ & 1.294 \\
\hline RSG-888 x ICC-4958 & $172.400 * *$ & 1.312 & $30.251 * *$ & $11.586^{* *}$ & 0.843 \\
\hline IPC-94-94 x RSG-888 & $580.800^{* *}$ & 1.300 & $494.347 * *$ & $32.892 *$ & 8.508 \\
\hline CSJD-901 x RSG-931 & $224.079 * *$ & 0.533 & $22.371 * *$ & $5.630 * *$ & 0.867 \\
\hline BG-362 x RSG-931 & $73.299 * *$ & 0.434 & $32.991 * *$ & $18.471 * *$ & 1.683 \\
\hline \multicolumn{6}{|l|}{ Plant height (cm) } \\
\hline RSG-895 x RSG-888 & $337.234 * *$ & 0.121 & $41.525 * *$ & $11.768 * *$ & 2.008 \\
\hline RSG-888 x ICC-4958 & $250.377 * *$ & 1.812 & $24.789 * *$ & $28.089 * *$ & 3.178 \\
\hline IPC-94-94 x RSG-888 & $43.056^{* *}$ & 0.936 & $86.982 * *$ & $20.519^{*}$ & 4.311 \\
\hline CSJD-901 x RSG-931 & $121.874 * *$ & 3.006 & $36.605^{* *}$ & $21.657^{* *}$ & 2.540 \\
\hline BG-362 x RSG-931 & $318.220^{* *}$ & 1.446 & $59.894 * *$ & $23.755^{*}$ & 5.010 \\
\hline \multicolumn{6}{|c|}{ Fruiting branches per plant } \\
\hline RSG-895 x RSG-888 & $137.217^{* *}$ & 0.565 & $17.083 * *$ & $6.903 * *$ & 0.776 \\
\hline RSG-888 x ICC-4958 & $27.950 * *$ & 0.983 & $18.536^{* *}$ & $6.496^{* *}$ & 1.073 \\
\hline IPC-94-94 x RSG-888 & $24.300 * *$ & 0.570 & $24.441 * *$ & $13.369 * *$ & 0.991 \\
\hline CSJD-901 x RSG-931 & $19.976^{* *}$ & 1.311 & $22.873 * *$ & $3.395^{*}$ & 0.738 \\
\hline BG-362 x RSG-931 & $11.371^{* *}$ & 0.099 & $19.045 * *$ & $4.941 * *$ & 0.441 \\
\hline \multicolumn{6}{|l|}{ Pods per plant } \\
\hline RSG-895 x RSG-888 & $973.674 * *$ & 7.163 & $419.413 * *$ & $86.127 * *$ & 9.789 \\
\hline RSG-888 x ICC-4958 & $650.164 * *$ & 3.330 & $341.484 * *$ & $64.771^{* *}$ & 7.821 \\
\hline IPC-94-94 x RSG-888 & $635.904 * *$ & 2.256 & $251.215^{* *}$ & $52.686^{* *}$ & 7.710 \\
\hline CSJD-901 x RSG-931 & $1152.828^{* *}$ & 8.139 & $224.393 * *$ & $68.672 * *$ & 10.861 \\
\hline BG-362 x RSG-931 & $555.212 * *$ & 2.795 & $231.613 * *$ & $43.422 * *$ & 8.834 \\
\hline \multicolumn{6}{|l|}{ Seeds per pod } \\
\hline RSG-895 x RSG-888 & $0.033^{*}$ & 0.011 & $0.071 * *$ & 0.004 & 0.006 \\
\hline RSG-888 x ICC-4958 & 0.033 & 0.004 & $0.150 * *$ & 0.003 & 0.007 \\
\hline IPC-94-94 x RSG-888 & $0.039^{*}$ & 0.001 & $0.069 * *$ & $0.016^{*}$ & 0.005 \\
\hline CSJD-901 x RSG-931 & $0.035^{*}$ & 0.006 & $0.066^{* *}$ & $0.015^{*}$ & 0.005 \\
\hline BG-362 x RSG-931 & 0.017 & 0.001 & $0.057 * *$ & 0.004 & 0.004 \\
\hline \multicolumn{6}{|c|}{ Biological yield per plant (g) } \\
\hline RSG-895 x RSG-888 & $191.572 * *$ & 2.206 & $60.081 * *$ & $17.504 * *$ & 2.564 \\
\hline
\end{tabular}




\begin{tabular}{|c|c|c|c|c|c|}
\hline RSG-888 x ICC-4958 & $385.137 * *$ & 0.936 & $111.629 * *$ & $38.121 * *$ & 2.276 \\
\hline IPC-94-94 x RSG-888 & $140.078^{* *}$ & 0.787 & $54.584 * *$ & $29.749 * *$ & 2.428 \\
\hline CSJD-901 x RSG-931 & $358.111 * *$ & 2.351 & $30.553 * *$ & $28.498 * *$ & 2.689 \\
\hline BG-362 x RSG-931 & $68.675^{* *}$ & 1.716 & $90.519 * *$ & $24.570^{* *}$ & 3.30 \\
\hline \multicolumn{6}{|l|}{ Seed yield per plant (g) } \\
\hline RSG-895 x RSG-888 & $172.777 * *$ & 1.125 & $25.269 * *$ & $10.524 * *$ & 0.970 \\
\hline RSG-888 x ICC-4958 & $23.870 * *$ & 0.726 & $62.193 * *$ & $6.248 * *$ & 1.004 \\
\hline IPC-94-94 x RSG-888 & $9.509 * *$ & 0.650 & $16.413 * *$ & $15.542 * *$ & 0.978 \\
\hline CSJD-901 x RSG-931 & $62.400 * *$ & 1.132 & $14.272 * *$ & $2.879 *$ & 0.606 \\
\hline BG-362 x RSG-931 & $19.018^{* *}$ & 0.414 & $51.234^{* *}$ & $4.280^{*}$ & 1.328 \\
\hline \multicolumn{6}{|l|}{ Harvest index (\%) } \\
\hline RSG-895 x RSG-888 & $231.778 * *$ & 5.879 & $96.939 * *$ & $37.386^{* *}$ & 4.537 \\
\hline RSG-888 x ICC-4958 & $29.489 * *$ & 1.553 & $123.058^{* *}$ & $25.721 * *$ & 1.600 \\
\hline IPC-94-94 x RSG-888 & $132.806^{* *}$ & 0.609 & $48.440 * *$ & $42.067 * *$ & 1.310 \\
\hline CSJD-901 x RSG-931 & $30.724 * *$ & 0.229 & $16.441 * *$ & $13.326^{* *}$ & 1.944 \\
\hline BG-362 x RSG-931 & $17.328 * *$ & 1.054 & $107.491 * *$ & $21.276^{* *}$ & 1.497 \\
\hline \multicolumn{6}{|l|}{ 100-seed weight (g) } \\
\hline RSG-895 x RSG-888 & $1.152 * *$ & 0.191 & $3.784 * *$ & $0.344 *$ & 0.077 \\
\hline RSG-888 x ICC-4958 & $14.658^{*}$ & 0.669 & $131.818^{* *}$ & 0.774 & 2.299 \\
\hline IPC-94-94 x RSG-888 & $8.175^{*}$ & 0.912 & $67.174 * *$ & 0.984 & 1.593 \\
\hline CSJD-901 x RSG-931 & $4.074 * *$ & 0.144 & $4.718^{* *}$ & $1.753^{*}$ & 0.394 \\
\hline BG-362 x RSG-931 & $3.931^{*}$ & 0.913 & $78.924 * *$ & 0.563 & 0.796 \\
\hline \multicolumn{6}{|l|}{ Protein content (\%) } \\
\hline RSG-895 x RSG-888 & $1.298 * *$ & 0.125 & $1.947 * *$ & 0.145 & 0.134 \\
\hline RSG-888 x ICC-4958 & $0.252 *$ & 0.035 & $3.075^{* *}$ & 0.111 & 0.045 \\
\hline IPC-94-94 x RSG-888 & $0.666^{* *}$ & 0.057 & $8.523^{* *}$ & $0.241^{*}$ & 0.078 \\
\hline CSJD-901 x RSG-931 & $1.285^{* *}$ & 0.113 & $2.610^{* *}$ & $0.823 * *$ & 0.109 \\
\hline BG-362 x RSG-931 & $2.191 * *$ & 0.037 & $1.288 * *$ & $0.505 * *$ & 0.104 \\
\hline
\end{tabular}

*, ** significant at 5 per cent and 1 per cent level, respectively

\subsection{Days to Maturity}

Main effects i.e., additive (d) and dominance (h) were significant in all the crosses under both the conditions. Among the two, the magnitudes of dominance effect prevailed over their respective additive gene effects in all the crosses except RSG-888 x ICC-4958 in irrigated and IPC-94-94 x RSG-888 in both irrigated and rainfed conditions, indicating importance of dominance effect in the inheritance of this trait. Among the digenic interactions, additive $\mathrm{x}$ additive (i) and dominance $\mathrm{x}$ dominance (l) interactions were significant in all the crosses under both the conditions except additive $x$ additive in RSG-895 $x$ RSG-888 and dominance $x$ dominance in IPC-94-94 x RSG-888 under rainfed. Higher magnitude of dominance $x$ dominance (1) than additive $x$ additive (i) interaction in all the crosses except IPC-94-94 x RSG-888 under both the conditions- indicated its importance in the inheritance of this trait. 
Table 3. Estimates of scaling test and gene effects for different characters in five chickpea crosses under irrigated (IRG) and rainfed (RF) conditions

\begin{tabular}{|c|c|c|c|c|c|c|c|c|c|c|}
\hline \multirow[t]{2}{*}{ Characters/crosses } & \multirow[t]{2}{*}{ Env. } & \multicolumn{2}{|l|}{ Scaling test } & \multirow{2}{*}{$-\chi^{2}(2)$} & \multirow{2}{*}{$\mathrm{m}$} & \multirow{2}{*}{ (d) } & \multirow{2}{*}{ (h) } & \multirow{2}{*}{ (i) } & \multirow{2}{*}{ (1) } & \multirow{2}{*}{ Epistasis } \\
\hline & & $\mathrm{C}$ & $\mathrm{D}$ & & & & & & & \\
\hline \multicolumn{11}{|c|}{ Days to 50\% flowering } \\
\hline \multirow[t]{2}{*}{$\begin{array}{l}\text { RSG-895 } \\
\text { RSG-888 }\end{array}$} & $\mathrm{x}_{\mathrm{IRG}}$ & $2.66 * \pm 1.16$ & $-6.40 * * \pm 1.44$ & $22.01 * *$ & $93.00 \pm 0.23$ & $-1.67 * * \pm 0.19$ & $9.38 * * \pm 1.01$ & $1.38 \pm 0.92$ & $-12.09 * * \pm 2.67$ & $\mathrm{D}$ \\
\hline & $\mathrm{RF}$ & $-2.94 * * \pm 0.68$ & $0.65 \pm 0.56$ & $19.32 * *$ & $83.17 \pm 0.11$ & $-1.50 * * \pm 0.12$ & $-3.45 * * \pm 0.41$ & $-3.92 * * \pm 0.44$ & $4.79 * * \pm 1.24$ & $\mathrm{D}$ \\
\hline \multirow[t]{2}{*}{$\begin{array}{l}\text { RSG- } 888 \\
\text { ICC-4958 }\end{array}$} & $\mathrm{x}_{\text {IRG }}$ & $0.00 \pm 0.69$ & $-6.00 * * \pm 0.85$ & $51.71 * *$ & $94.00 \pm 0.14$ & $-2.67 * * \pm 0.13$ & $4.67^{* *} \pm 0.58$ & $-1.33^{*} \pm 0.56$ & $-8.00 * * \pm 1.57$ & $\mathrm{D}$ \\
\hline & $\mathrm{RF}$ & $-5.00 * * \pm 0.76$ & $5.66^{* *} \pm 0.72$ & $87.84 * *$ & $87.00 \pm 0.14$ & $-2.50 * * \pm 0.11$ & $-5.77 * * \pm 0.52$ & $-9.61 * * \pm 0.52$ & $14.21 * * \pm 1.53$ & $\mathrm{D}$ \\
\hline \multirow[t]{2}{*}{$\begin{array}{l}\text { IPC-94-94 } \\
\text { RSG-888 }\end{array}$} & $\mathrm{x}_{\mathrm{IRG}}$ & $20.01 * * \pm 1.34$ & $21.32 * * \pm 0.98$ & $1072.27 * *$ & $* 87.67 \pm 0.30$ & $-12.67 * * \pm 0.24$ & $4-2.21 * * \pm 0.73$ & $-36.21 * * \pm 0.90$ & $01.75 \pm 2.56$ & - \\
\hline & $\mathrm{RF}$ & $-24.35 * * \pm 1.17$ & $7-14.99 * * \pm 1.14$ & $842.24 * *$ & $66.33 \pm 0.26$ & $-11.17 * * \pm 0.20$ & $00.44 \pm 0.82$ & $-16.40 * * \pm 0.88$ & $812.48 * * \pm 2.48$ & - \\
\hline \multirow[t]{2}{*}{$\begin{array}{l}\text { CSJD-901 } \\
\text { RSG-931 }\end{array}$} & ${ }^{\mathrm{x}}$ IRG & $2.28 * * \pm 0.72$ & $-1.79 * * \pm 0.58$ & $17.45 * *$ & $91.90 \pm 0.12$ & $-1.00 * * \pm 0.14$ & $3.57 * * \pm 0.42$ & $-0.43 \pm 0.48$ & $-5.43 * * \pm 1.30$ & $\mathrm{D}$ \\
\hline & RF & $3.00 * * \pm 0.68$ & $-7.33 * * \pm 0.57$ & $176.82 * *$ & $85.83 \pm 0.11$ & $-1.83 * * \pm 0.13$ & $3.88 * * \pm 0.40$ & $1.72 * * \pm 0.46$ & $-13.78 * * \pm 1.24$ & $\mathrm{D}$ \\
\hline \multirow[t]{2}{*}{ BG-362 x RSG-931 } & IRG & $3.67 * * \pm 0.74$ & $-4.99 * * \pm 0.73$ & $57.52 * *$ & $95.33 \pm 0.16$ & $2.17 * * \pm 0.11$ & $3.77 * * \pm 0.53$ & $8.28 * * \pm 0.53$ & $-11.54 * * \pm 1.55$ & $\mathrm{D}$ \\
\hline & $\mathrm{RF}$ & $7.35 * * \pm 0.66$ & $2.01^{* *} \pm 0.57$ & $144.48 * *$ & $93.67 \pm 0.11$ & $3.34 * * \pm 0.13$ & $2.22 * * \pm 0.40$ & $6.55 * * \pm 0.45$ & $-7.12 * * \pm 1.22$ & $\mathrm{D}$ \\
\hline \multicolumn{11}{|l|}{ Days to maturity } \\
\hline \multirow[t]{2}{*}{$\begin{array}{l}\text { RSG-895 } \\
\text { RSG-888 }\end{array}$} & ${ }^{\mathrm{x}}$ IRG & $4.02 * * \pm 1.86$ & $-6.65 * * \pm 1.33$ & $25.35 * *$ & $138.67 \pm 0.43$ & $31.33 * * \pm 0.23$ & $8.44 * * \pm 1.06$ & $7.77 * * \pm 1.22$ & $-14.22 * * \pm 3.70$ & $\mathrm{D}$ \\
\hline & $\mathrm{RF}$ & $0.34 \pm 1.14$ & $-6.34 * * \pm 1.13$ & $31.91 * *$ & $130.67 \pm 0.23$ & $3-1.83 * * \pm 0.20$ & $8.45^{* *} \pm 0.81$ & $0.62 \pm 0.82$ & $-8.91 * * \pm 2.34$ & $\mathrm{D}$ \\
\hline \multirow[t]{2}{*}{$\begin{array}{l}\text { RSG-888 } \\
\text { ICC-4958 }\end{array}$} & $\mathrm{x}_{\mathrm{IRG}}$ & $1.00 \pm 1.16$ & $-7.67 * * \pm 1.12$ & $47.22 * *$ & $138.00 \pm 0.22$ & $2-3.83 * * \pm 0.23$ & $3.78 * * \pm 0.78$ & $-2.38 * * \pm 0.83$ & $-11.56^{* *} \pm 0.29$ & $\mathrm{D}$ \\
\hline & $\mathrm{RF}$ & $2.12 \pm 1.14$ & $-2.72 * \pm 1.09$ & $8.14^{*}$ & $134.03 \pm 0.23$ & $3-2.00 * * \pm 0.20$ & $6.51 * * \pm 0.78$ & $-1.83^{*} \pm 0.81$ & $-6.45^{* *} \pm 2.31$ & $\mathrm{D}$ \\
\hline \multirow[t]{2}{*}{$\begin{array}{l}\text { IPC-94-94 } \\
\text { RSG-888 }\end{array}$} & $\mathrm{x}_{\mathrm{IRG}}$ & $7.66^{* * \pm 2.37}$ & $16.34 * * \pm 1.98$ & $101.67 * *$ & $125.00 \pm 0.50$ & $0-12.50 * * \pm 0.41$ & $1-7.79 * * \pm 1.45$ & $-34.61 * * \pm 1.67$ & $711.57 * \pm 4.66$ & $\mathrm{D}$ \\
\hline & $\mathrm{RF}$ & $-10.99 * * \pm 1.46$ & $6-15.01 * * \pm 1.34$ & $240.08 * *$ & $114.00 \pm 0.29$ & $9-13.17 * * 0.20$ & $06.68 * * \pm 0.99$ & $-18.17 * * \pm 1.00$ & $0-5.35 \pm 2.95$ & - \\
\hline \multirow[t]{2}{*}{$\begin{array}{l}\text { CSJD-901 } \\
\text { RSG-931 }\end{array}$} & $\mathrm{x}_{\mathrm{IRG}}$ & $5.01 * * \pm 1.08$ & $-0.34 \pm 1.10$ & $21.46^{* *}$ & $134.67 \pm 0.20$ & $01.50 * * \pm 0.22$ & $4.89 * * \pm 0.76$ & $4.06 * * \pm 0.79$ & $-7.12 * * \pm 2.17$ & $\mathrm{D}$ \\
\hline & $\mathrm{RF}$ & $2.99 * *_{ \pm} 1.11$ & $-0.35 \pm 1.08$ & $7.24 * *$ & $129.00 \pm 0.20$ & $0-1.17 * * \pm 0.21$ & $5.57 * * \pm 0.76$ & $-1.60 * \pm 0.78$ & $-4.45 * \pm 2.18$ & $\mathrm{D}$ \\
\hline \multirow[t]{2}{*}{ BG-362 x RSG-931 } & IRG & $-0.96 \pm 1.17$ & $4.38 * * \pm 1.30$ & $11.56^{* *}$ & $134.00 \pm 0.22$ & $24.52 * * \pm 0.22$ & $-4.90 * * \pm 0.90$ & $5.95 * * \pm 0.87$ & $7.12 * * \pm 2.47$ & $\mathrm{D}$ \\
\hline & $\mathrm{RF}$ & $3.64 * * \pm 1.18$ & $10.32 * * \pm 1.13$ & $101.76^{* *}$ & $132.33 \pm 0.23$ & $31.50 * * \pm 0.25$ & $-1.77 * \pm 0.78$ & $-3.27 * * \pm 0.82$ & $8.91 * * \pm 2.30$ & $\mathrm{D}$ \\
\hline \multicolumn{11}{|l|}{ Plant height (cm) } \\
\hline \multirow[t]{2}{*}{$\begin{array}{l}\text { RSG-895 } \\
\text { RSG-888 }\end{array}$} & $\mathrm{x}_{\mathrm{IRG}}$ & $7.90 \pm 4.58$ & $-14.64 * * \pm 4.65$ & $10.92 * *$ & $61.00 \pm 0.98$ & $2.05 * * \pm 0.79$ & $17.29 * * \pm 3.31$ & $15.18 * * \pm 3.37$ & $-30.05 * * \pm 9.69$ & $\mathrm{D}$ \\
\hline & $\mathrm{RF}$ & $0.40 \pm 3.47$ & $-10.04 * * \pm 2.77$ & $13.77 * *$ & $52.33 \pm 0.66$ & $-1.20 \pm 0.53$ & $10.16^{* *} \pm 2.08$ & $4.36 \pm 2.26$ & $-13.92 * \pm 6.61$ & $\mathrm{D}$ \\
\hline \multirow[t]{2}{*}{$\begin{array}{l}\text { RSG-888 } \\
\text { ICC-4958 }\end{array}$} & $\mathrm{x}_{\mathrm{IRG}}$ & $6.92 \pm 4.11$ & $11.02 * * \pm 4.06$ & $11.13 * *$ & $59.23 \pm 0.73$ & $-4.47 * * \pm 0.84$ & $-6.39 * \pm 2.81$ & $-15.13^{* *} \pm 2.82$ & $25.47 \pm 8.04$ & - \\
\hline & $\mathrm{RF}$ & $0.47 \pm 3.34$ & $13.49 * * \pm 2.93$ & $23.76^{* *}$ & $52.82 \pm 0.71$ & $1.13^{*} \pm 0.52$ & $-4.98 * \pm 2.16$ & $-6.64 * * \pm 2.29$ & $17.36 * \pm 6.74$ & $\mathrm{D}$ \\
\hline $\begin{array}{l}\text { IPC-94-94 } \\
\text { RSG-888 }\end{array}$ & $\mathrm{x}_{\mathrm{IRG}}$ & $7.51 \pm 4.08$ & $10.47 * * \pm 3.57$ & $15.55 * *$ & $50.23 \pm 0.84$ & $-5.57 * * \pm 0.72$ & $-5.69 * \pm 2.58$ & $-16.86^{* * *} \pm 2.84$ & $43.95 \pm 8.05$ & - \\
\hline & $\mathrm{RF}$ & $-1.54 \pm 3.59$ & $-15.04 * * \pm 2.68$ & $38.32 * *$ & $48.05 \pm 0.76$ & $-4.90 * * \pm 0.54$ & $13.30 * * \pm 2.06$ & $-0.03 \pm 2.35$ & $-18.00 * * \pm 6.92$ & $\mathrm{D}$ \\
\hline $\begin{array}{l}\text { CSJD-901 } \\
\text { RSG-931 }\end{array}$ & $\mathrm{x}_{\mathrm{IRG}}$ & $4.73 \pm 4.34$ & $-12.25 * * \pm 3.53$ & $12.22 * *$ & $56.45 \pm 0.96$ & $-2.60 * * \pm 0.46$ & $15.22 * * \pm 2.75$ & $3.75 \pm 2.91$ & $-22.64 * \pm 8.89$ & $\mathrm{D}$ \\
\hline & $\mathrm{RF}$ & $4.48 \pm 3.11$ & $11.83 * * \pm 2.88$ & $18.46^{* *}$ & $50.92 \pm 0.51$ & $0.93 \pm 0.80$ & $-2.54 \pm 1.86$ & $-5.28 * \pm 2.37$ & $9.80 \pm 5.52$ & - \\
\hline BG-362 x RSG-931 & IRG & $2.98 \pm 3.81$ & $16.38 * * \pm 3.63$ & $23.45 * *$ & $60.45 \pm 0.76$ & $2.05^{* *} \pm 0.67$ & $-5.44 * \pm 2.60$ & $-6.32 * \pm 2.69$ & $17.87 * \pm 7.71$ & $\mathrm{D}$ \\
\hline & $\mathrm{RF}$ & $4.38 \pm 3.48$ & $-11.36^{* *} \pm 3.25$ & $12.34 * *$ & $55.18 \pm 0.71$ & $5.57 * * \pm 0.54$ & $10.67^{* *} \pm 2.37$ & $19.44 * * \pm 2.41$ & $-20.99 * * \pm 7.10$ & $\mathrm{D}$ \\
\hline $\begin{array}{l}\text { Fruiting branch } \\
\text { plant }\end{array}$ & es pe & & & & & & & & & \\
\hline $\begin{array}{l}\text { RSG-895 } \\
\text { RSG-888 }\end{array}$ & $\mathrm{x}_{\mathrm{IRG}}$ & $6.42 * * \pm 2.38$ & $-3.92 \pm 2.60$ & $8.37 *$ & $17.68 \pm 0.47$ & $-1.25 * * \pm 0.41$ & $8.19^{* * \pm 1.84}$ & $1.18 \pm 1.79$ & $-13.79^{* *} \pm 5.10$ & $\mathrm{D}$ \\
\hline & $\mathrm{RF}$ & $0.63 \pm 1.81$ & $8.33^{* *} \pm 2.14$ & $16.01 * *$ & $11.28 \pm 0.38$ & $-1.72 * * \pm 0.37$ & $-4.23 * * \pm 1.47$ & $-8.88 * * \pm 1.46$ & $10.27 * \pm 4.00$ & $\mathrm{D}$ \\
\hline
\end{tabular}




\begin{tabular}{|c|c|c|c|c|c|c|c|c|c|c|}
\hline \multirow[t]{2}{*}{$\begin{array}{l}\text { RSG-888 } \\
\text { ICC-4958 }\end{array}$} & $\mathrm{x}_{\mathrm{IRG}}$ & $3.42 \pm 2.34$ & $13.38 * * \pm 2.72$ & $27.63 * *$ & $16.67 \pm 0.44$ & $1.53 * * \pm 0.52$ & $-4.32 * \pm 1.84$ & $-5.29 * * \pm 1.89$ & $13.28 * * \pm 4.96$ & $\mathrm{D}$ \\
\hline & $\mathrm{RF}$ & $-0.14 \pm 2.37$ & $10.07 * * \pm 2.00$ & $25.91 * *$ & $13.97 \pm 0.45$ & $-1.00 * \pm 0.48$ & $-4.80 * * \pm 1.40$ & $-8.73 * * \pm 1.58$ & $13.62 * * \pm 4.43$ & $\mathrm{D}$ \\
\hline \multirow[t]{2}{*}{$\begin{array}{l}\text { IPC-94-94 } \\
\text { RSG-888 }\end{array}$} & ${ }^{x}$ IRG & $2.26 \pm 2.89$ & $-11.88 * * \pm 2.33$ & $26.54 * *$ & $16.78 \pm 0.57$ & $-2.07 * * \pm 0.42$ & $12.93 * * \pm 1.76$ & $4.16^{*} \pm 1.90$ & $-18.85^{* *} \pm 5.59$ & $\mathrm{D}$ \\
\hline & $\mathrm{RF}$ & $3.10 \pm 2.51$ & $12.32 * * \pm 2.17$ & $35.54 * *$ & $13.62 \pm 0.42$ & $-1.45 * * \pm 0.46$ & $-5.05 * * \pm 1.55$ & $-10.60 * * \pm 1.71$ & $112.29 * * \pm 4.65$ & D \\
\hline \multirow[t]{2}{*}{$\begin{array}{l}\text { CSJD-901 } \\
\text { RSG-931 }\end{array}$} & $\mathrm{x}_{\mathrm{IRG}}$ & $0.32 \pm 2.95$ & $-8.19^{* *} \pm 2.80$ & $8.76^{*}$ & $16.43 \pm 0.60$ & $-1.97 * * \pm 0.58$ & $10.08 * * \pm 1.97$ & $1.58 \pm 2.09$ & $-11.35 \pm 5.92$ & 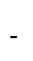 \\
\hline & $\mathrm{RF}$ & $5.32 * \pm 2.41$ & $-4.46 * \pm 2.05$ & $7.28 *$ & $15.13 \pm 0.51$ & $-0.77 * \pm 0.36$ & $6.06^{* *} \pm 1.53$ & $2.32 \pm 1.63$ & $-13.04 * * \pm 4.84$ & $\mathrm{D}$ \\
\hline \multirow[t]{2}{*}{ BG-362 x RSG-931 } & IRG & $3.35 \pm 2.97$ & $13.68 * * \pm 2.58$ & $35.43 * *$ & $17.32 \pm 0.63$ & $-1.20 * \pm 0.50$ & $-4.60 * \pm 1.88$ & $-10.96 * * \pm 2.06$ & $613.78 * \pm 5.92$ & $\mathrm{D}$ \\
\hline & $\mathrm{RF}$ & $-0.39 \pm 3.14$ & $9.87 * * \pm 2.55$ & $16.34 * *$ & $15.37 \pm 0.68$ & $0.97 \pm 0.53$ & $-4.38 * \pm 1.88$ & $-4.71 * \pm 2.20$ & $13.68 * \pm 6.17$ & $\mathrm{D}$ \\
\hline \multicolumn{11}{|l|}{ Pods per plant } \\
\hline \multirow[t]{2}{*}{$\begin{array}{l}\text { RSG-895 } \\
\text { RSG-888 }\end{array}$} & ${ }^{\mathrm{x}}$ IRG & $20.76 \pm 10.69$ & $-45.60 * * \pm 11.82$ & $216.02 * *$ & $62.75 \pm 2.30$ & $-7.15 * * \pm 1.79$ & $50.08 * * \pm 8.37$ & $19.56 * \pm 8.23$ & $-88.48 * * \pm 23.60$ & \\
\hline & $\mathrm{RF}$ & $34.12 * * \pm 7.43$ & $8.60 \pm 8.64$ & $26.99 * *$ & $50.63 \pm 1.67$ & $-2.30 * \pm 0.95$ & $13.81 * \pm 6.21$ & $-4.65 \pm 5.81$ & $-34.03 * \pm 17.24$ & $\mathrm{D}$ \\
\hline \multirow{2}{*}{$\begin{array}{l}\text { RSG-888 } \\
\text { ICC-4958 }\end{array}$} & $\mathrm{x}_{\mathrm{IRG}}$ & $11.76 \pm 7.09$ & $41.66^{* * \pm 8.32}$ & $34.21 * *$ & $63.28 \pm 1.52$ & $3.68 * * \pm 0.93$ & $-15.63 * * \pm 5.95$ & $5-18.45 * * \pm 5.4$ & $939.87 * \pm 16.29$ & $\mathrm{D}$ \\
\hline & $\mathrm{RF}$ & $21.71 * * \pm 9.07$ & $52.39 * * \pm 8.98$ & $55.66 * *$ & $55.72 \pm 2.02$ & $-4.99 * * \pm 1.12$ & $-17.49 * * \pm 6.64$ & $4-41.28 * * \pm 6$ & $840.91 * \pm 19.71$ & $\mathrm{D}$ \\
\hline \multirow[t]{2}{*}{$\begin{array}{l}\text { IPC-94-94 } \\
\text { RSG-888 }\end{array}$} & ${ }^{x}$ IRG & $8.95 \pm 8.26$ & $37.11 * * \pm 7.23$ & $37.50 * *$ & $60.17 \pm 1.86$ & $-4.97 * * \pm 1.07$ & $-12.05 * * \pm 5.46$ & $6-33.18 * * \pm 5$ & $937.55 * \pm 17.23$ & $\mathrm{D}$ \\
\hline & $\mathrm{RF}$ & $5.74 \pm 6.93$ & $33.74 * * \pm 6.99$ & $29.38 * *$ & $51.00 \pm 1.53$ & $3.20 * * \pm 0.94$ & $-7.47 \pm 5.11$ & $-15.14 * * \pm 5.01$ & $137.33 * \pm 15.04$ & - \\
\hline \multirow{2}{*}{$\begin{array}{l}\text { CSJD-901 } \\
\text { RSG-931 }\end{array}$} & $\mathrm{x}_{\mathrm{IRG}}$ & $4.35 \pm 8.92$ & $-27.15 * * \pm 8.84$ & $9.78 * *$ & $61.37 \pm 1.98$ & $-2.53 * \pm 1.07$ & $30.06 * * \pm 6.54$ & $13.76 * \pm 6.44$ & $-42.00 * \pm 19.38$ & $\mathrm{D}$ \\
\hline & $\mathrm{RF}$ & $52.08 * * \pm 9.40$ & $-0.46 \pm 7.94$ & $35.80 * *$ & $55.67 \pm 2.09$ & $-3.17 * \pm 1.24$ & $18.09 * * \pm 6.03$ & $2.65 \pm 6.39$ & $-70.05 * * \pm 19.29$ & $9 \mathrm{D}$ \\
\hline \multirow[t]{2}{*}{ BG-362 x RSG-931 } & IRG & $7.99 \pm 8.96$ & $38.71 * * \pm 8.14$ & $31.70 * *$ & $61.58 \pm 2.00$ & $-3.47 * * \pm 0.89$ & $-14.04 * \pm 6.20$ & $-31.40 * * \pm 6.18$ & $840.96 * \pm 19.05$ & $\mathrm{D}$ \\
\hline & $\mathrm{RF}$ & $1.21 \pm 13.96$ & $45.51 * * \pm 10.15$ & $27.28 * *$ & $51.52 \pm 3.23$ & $4.04 * \pm 1.80$ & $-19.23 * \pm 8.06$ & $-22.07 * \pm 9.16$ & $59.07 * \pm 27.95$ & $\mathrm{D}$ \\
\hline \multicolumn{11}{|l|}{ Seeds per pod } \\
\hline $\begin{array}{l}\text { RSG-895 } \\
\text { RSG-888 }\end{array}$ & \multicolumn{2}{|c|}{$\mathrm{x}_{\text {Pooled }-0.02 \pm 0.20}$} & $-0.86 * * \pm 0.22$ & $15.66 * *$ & $1.72 \pm 0.04$ & $0.07 \pm 0.04$ & $0.58 * * \pm 0.15$ & $0.71 * * \pm 0.15$ & $-1.12 * * \pm 0.41$ & $\mathrm{D}$ \\
\hline $\begin{array}{l}\text { RSG-888 } \\
\text { ICC-4958 }\end{array}$ & \multicolumn{2}{|c|}{$\mathrm{x}_{\text {Pooled } 0.01 \pm 0.24}$} & $-0.74 * * \pm 0.21$ & $14.24 * *$ & $1.51 \pm 0.05$ & $0.19 * * \pm 0.04$ & $0.51 * * \pm 0.15$ & $0.88^{* *} \pm 0.16$ & $-1.00 * \pm 0.48$ & D \\
\hline \multirow{2}{*}{$\begin{array}{l}\text { IPC-94-94 } \\
\text { RSG-888 }\end{array}$} & ${ }^{\mathrm{x}} \mathrm{IRG}$ & $-0.10 \pm 0.41$ & $0.94 * \pm 0.38$ & $6.21 *$ & $1.67 \pm 0.09$ & $-0.05 \pm 0.07$ & $-0.49 \pm 0.28$ & $-0.74 * \pm 0.29$ & $1.39 \pm 0.85$ & - \\
\hline & $\mathrm{RF}$ & $0.28 \pm 0.30$ & $0.60 * \pm 0.30$ & $6.06^{*}$ & $1.65 \pm 0.07$ & $-0.12 * \pm 0.05$ & $-0.41 \pm 0.22$ & $-0.59 * * \pm 0.22$ & $0.43 \pm 0.64$ & - \\
\hline \multirow{2}{*}{$\begin{array}{l}\text { CSJD-901 } \\
\text { RSG-931 }\end{array}$} & $\mathrm{x}_{\mathrm{IRG}}$ & $0.10 \pm 0.32$ & $-0.86 * * \pm 0.33$ & $6.86^{* *}$ & $1.79 \pm 0.07$ & $0.15 * * \pm 0.05$ & $0.48 * \pm 0.24$ & $0.89 * * \pm 0.23$ & $-1.28 \pm 0.68$ & - \\
\hline & $\mathrm{RF}$ & $0.06 \pm 0.26$ & $-0.70 * \pm 0.28$ & $6.28 *$ & $1.72 \pm 0.05$ & $0.03 \pm 0.06$ & $0.48 * \pm 0.19$ & $0.52 * \pm 0.21$ & $-1.01 \pm 0.54$ & - \\
\hline BG-362 x RSG-931 & Poole & $d-0.09 \pm 0.22$ & $-0.70 * * \pm 0.19$ & $14.10 * *$ & $1.66 \pm 0.04$ & $0.07 \pm 0.04$ & $0.35^{*} \pm 0.14$ & $0.58 * * \pm 0.15$ & $-0.80 \pm 0.42$ & \\
\hline
\end{tabular}

\section{Biological yield per plant}

(g)

RSG-895

RSG-888

${ }^{\mathrm{x}} \mathrm{IRG} \quad 2.96 \pm 5.47$

$-18.80 * * \pm 5.9010 .19 * *$

$41.11 \pm 1.11-2.41 * * \pm 0.86 \quad 19.11^{* *} \pm 4.21 \quad 8.21 * \pm 4.11 \quad-29.01 * \pm 11.83 \quad \mathrm{D}$

RF $\quad 2.94 \pm 5.11$

$-14.62 * * \pm 5.556 .95 *$

$35.68 \pm 1.02 \quad 2.32 * * \pm 0.80 \quad 15.23 * * \pm 3.95 \quad 14.88 * * \pm 3.78-23.41 * \pm 11.03 \mathrm{D}$

RSG-888

ICC-4958

$\mathrm{x}_{\mathrm{IRG}} \quad 7.76 \pm 5.94$

$-18.16^{* *} \pm 6.688 .27 *$

$48.32 \pm 1.09-2.79 * * \pm 1.01 \quad 21.76 * * \pm 4.697 .82 \pm 4.47 \quad-34.56 * * \pm 12.71 \mathrm{D}$

RF $\quad 9.19 \pm 5.11$

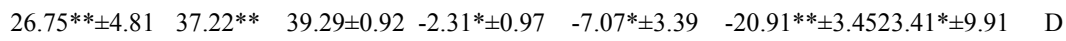

IPC-94-94

${ }_{\text {IRG }} \quad 8.97 \pm 4.71$

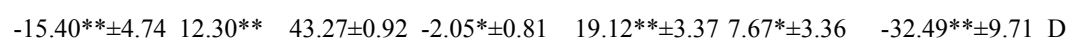

RSG-888

RF $\quad 0.61 \pm 4.47 \quad 15.27 * * \pm 3.41 \quad 20.83 * * \quad 35.82 \pm 0.71 \quad 2.02 * * \pm 0.62 \quad-3.59 \pm 2.60 \quad-6.05^{*} \pm 2.67 \quad 19.55^{*} \pm 8.06 \quad-$

CSJD-901

RSG-931

$\mathrm{x}_{\mathrm{IRG}} \quad-5.11 \pm 5.75$

$16.39 * * \pm 4.89 \quad 11.35 * * \quad 38.11 \pm 1.25 \quad-1.88 * \pm 0.81 \quad-5.79 \pm 3.68 \quad-15.55 * * \pm 4.0028 .67 * \pm 11.67 \quad-$

RF $\quad 13.46 \pm 4.81 \quad-20.96 * * \pm 3.84 \quad 31.50 * * \quad 35.52 \pm 0.95 \quad-2.73 * * \pm 0.69 \quad 16.89 * * \pm 2.91 \quad 10.76 * * \pm 3.09 \quad-45.89 * * \pm 9.28 \quad \mathrm{D}$

BG-362 x RSG-931 IRG $17.69 * * \pm 5.97 \quad 27.89 * * \pm 5.88 \quad 41.49 * * \quad 44.46 \pm 1.25 \quad-2.19 * \pm 0.90 \quad-9.20 * \pm 4.27 \quad-20.01 * * \pm 4.2713 .60 \pm 12.55 \quad-$

RF $\quad-0.49 \pm 7.39 \quad 23.37 * * \pm 5.09 \quad 25.21 * * \quad 38.27 \pm 1.59 \quad 2.49 * \pm 1.05 \quad-10.61 * * \pm 4.03-10.69 * \pm 4.73 \quad 31.81 * \pm 14.11 \quad \mathrm{D}$

Seed yield per plant $(g)$

RSG-895 $\quad x I R G \quad 2.91 \pm 2.97 \quad 13.13 * * \pm 3.26 \quad 21.11 * * \quad 18.63 \pm 0.66 \quad-1.64 * * \pm 0.42 \quad-3.79 \pm 2.35 \quad-11.56 * * \pm 2.2713 .63 * \pm 6.66 \quad-$ 
RSG-888

\begin{tabular}{|c|c|c|c|c|c|c|c|c|c|c|}
\hline & RF & $1.10 \pm 2.53$ & $-9.88 * * \pm 2.68$ & $13.63 * *$ & $14.60 \pm 0.48$ & $-1.05 * \pm 0.49$ & $10.82 * * \pm 1.86$ & $4.67 * \pm 1.88$ & $-14.64 * * \pm 5.21$ & $\mathrm{D}$ \\
\hline \multirow[t]{2}{*}{$\begin{array}{l}\text { RSG- } 888 \\
\text { ICC-4958 }\end{array}$} & $\mathrm{x}_{\mathrm{IRG}}$ & $4.61 \pm 3.08$ & $14.83^{* *} \pm 3.17$ & $29.35^{* *}$ & $20.73 \pm 0.67$ & $1.14 * \pm 0.53$ & $-3.39 \pm 2.26$ & $-6.85 * * 2.35$ & $13.63 * \pm 6.59$ & \\
\hline & $\mathrm{RF}$ & $6.44 \pm 3.30$ & $19.04 * * \pm 3.13$ & $41.17^{* *}$ & $19.19 \pm 0.57$ & $-1.60 * \pm 0.77$ & $-5.00 * \pm 2.09$ & $-14.82 * * \pm 2.51$ & $116.80 * * \pm 6.12$ & $\mathrm{D}$ \\
\hline \multirow{2}{*}{$\begin{array}{l}\text { IPC-94-94 } \\
\text { RSG-888 }\end{array}$} & $\mathrm{x}_{\mathrm{IRG}}$ & $2.43 \pm 2.34$ & $-7.65 * * \pm 2.56$ & $9.37 * *$ & $19.08 \pm 0.47$ & $-1.28 * * \pm 0.49$ & $9.04 * * \pm 1.76$ & $2.95 \pm 1.78$ & $-13.44 * * \pm 4.92$ & \\
\hline & $\mathrm{RF}$ & $0.35 \pm 2.74$ & $13.47 * * \pm 2.21$ & $37.95 * *$ & $16.63 \pm 0.43$ & $1.93^{* *} \pm 0.44$ & $-4.75^{* *} \pm 1.63$ & $-5.07 * * \pm 1.68$ & $17.49 * * \pm 4.97$ & $\mathrm{D}$ \\
\hline \multirow{2}{*}{$\begin{array}{l}\text { CSJD-901 } \\
\text { RSG-931 }\end{array}$} & $\mathrm{x}_{\mathrm{IRG}}$ & $-6.70 * \pm 3.22$ & $-4.45 \pm 2.96$ & $9.83 * *$ & $15.09 \pm 0.72$ & $-1.11 * \pm 0.49$ & $5.32 * \pm 2.18$ & $-0.36 \pm 2.31$ & $3.00 \pm 6.74$ & \\
\hline & $\mathrm{RF}$ & $5.46 * \pm 2.75$ & $-7.36^{* *} \pm 2.17$ & $12.55 * *$ & $14.23 \pm 0.55$ & $-0.96 * \pm 0.38$ & $7.61 * * \pm 1.66$ & $3.90 * \pm 0.74$ & $-17.09 * *_{ \pm} 5.31$ & $\mathrm{D}$ \\
\hline \multirow[t]{2}{*}{ BG-362 x RSG-931 } & 1 IRG & $6.20 * \pm 2.85$ & $15.73 * * \pm 2.97$ & $42.01 * *$ & $20.00 \pm 0.61$ & $-0.92 * \pm 0.41$ & $-4.46^{*} \pm 2.15$ & $-11.28 * * \pm 2.09$ & $912.70 * \pm 6.18$ & \\
\hline & $\mathrm{RF}$ & $5.01 \pm 3.91$ & $17.21^{* *} \pm 2.90$ & $51.77 * *$ & $18.21 \pm 0.86$ & $1.45 * \pm 0.56$ & $-5.09 * \pm 2.25$ & $-7.75^{* *} \pm 2.57$ & $16.27 * \pm 7.65$ & \\
\hline \multicolumn{11}{|l|}{ Harvest index (\%) } \\
\hline \multirow[t]{2}{*}{$\begin{array}{l}\text { RSG-895 } \\
\text { RSG-888 }\end{array}$} & $\mathrm{x}_{\mathrm{IRG}}$ & $4.83 \pm 8.00$ & $30.19^{* *} \pm 6.74$ & $28.43 * *$ & $46.45 \pm 1.83$ & $-2.82 * * \pm 0.81$ & $-12.54 * * \pm 5$ & $3-24.97 * * \pm 5.44$ & $433.82 * \pm 16.74$ & \\
\hline & $\mathrm{RF}$ & $5.24 \pm 5.78$ & $-13.26 * * \pm 4.97$ & $7.17 *$ & $43.35 \pm 1.17$ & $-3.61 * * \pm 0.90$ & $17.91 * * \pm 3.67$ & $2.49 \pm 3.90$ & $-24.67 * \pm 11.43$ & $\mathrm{D}$ \\
\hline \multirow[t]{2}{*}{$\begin{array}{l}\text { RSG- } 888 \\
\text { ICC-4958 }\end{array}$} & $\mathrm{x}_{\text {IRG }}$ & $8.66 \pm 7.23$ & $30.05 * * \pm 6.60$ & $31.37 * *$ & $47.85 \pm 1.68$ & $3.17^{* *} \pm 0.96$ & $-11.47^{*} \pm 4.94$ & $-12.26 * \pm 5.09$ & $28.52 \pm 15.40$ & \\
\hline & $\mathrm{RF}$ & $7.13 \pm 5.05$ & $24.05 * * \pm 4.77$ & $30.47 * *$ & $50.12 \pm 1.02$ & $-2.50 * \pm 1.06$ & $-6.04 \pm 3.30$ & $-19.84 * *_{ \pm} 3.52$ & $222.56 * \pm 9.97$ & - \\
\hline \multirow[t]{2}{*}{$\begin{array}{l}\text { IPC-94-94 } \\
\text { RSG-888 }\end{array}$} & $\mathrm{x}_{\mathrm{IRG}}$ & $7.47 \pm 5.13$ & $-15.05^{* *} \pm 5.82$ & $7.23^{*}$ & $47.08 \pm 1.11$ & $-1.39 * \pm 0.56$ & $16.21 * * \pm 4.22$ & $8.49 * \pm 3.92$ & $-30.03 * \pm 11.73$ & \\
\hline & $\mathrm{RF}$ & $4.15 \pm 3.78$ & $23.37 * * \pm 4.50$ & $27.48^{* *}$ & $49.17 \pm 0.66$ & $3.18 * * \pm 1.10$ & $-7.39 * * \pm 2.84$ & $-8.54 * * 3.15$ & $25.63 * *_{ \pm} 7.51$ & $\mathrm{D}$ \\
\hline \multirow[t]{2}{*}{$\begin{array}{l}\text { CSJD-901 } \\
\text { RSG-931 }\end{array}$} & $\mathrm{x}_{\mathrm{IRG}}$ & $2.80 \pm 3.81$ & $-8.56 * * \pm 3.26$ & $7.67^{*}$ & $44.24 \pm 0.63$ & $-0.90 \pm 0.98$ & $10.73^{* *} \pm 2.09$ & $4.37 \pm 2.66$ & $-15.15^{* *} \pm 6.50$ & \\
\hline & $\mathrm{RF}$ & $-2.52 \pm 3.77$ & $12.22 * * \pm 3.26$ & $14.04 * *$ & $40.06 \pm 0.78$ & $1.61 * \pm 0.81$ & $-5.25^{*} \pm 2.26$ & $-5.35^{*} \pm 2.56$ & $19.65 * * \pm 7.23$ & $\mathrm{D}$ \\
\hline \multirow[t]{2}{*}{ BG-362 x RSG-931 } & I IRG & $3.99 \pm 5.02$ & $16.89 * * \pm 3.95$ & $20.91 * *$ & $46.02 \pm 0.94$ & $-1.65 \pm 0.92$ & $-3.78 \pm 2.87$ & $-13.89 * * \pm 3.35$ & $517.21 \pm 9.26$ & \\
\hline & $\mathrm{RF}$ & $11.65 * * \pm 3.71$ & $25.37 * * \pm 3.28$ & $78.62 * *$ & $48.86 \pm 0.68$ & $3.03 * * \pm 0.69$ & $-5.47 * \pm 2.34$ & $-8.92 * * \pm 2.55$ & $18.29 * * \pm 7.08$ & . \\
\hline
\end{tabular}

\begin{tabular}{|c|c|c|c|c|c|c|c|c|c|c|}
\hline \multirow[t]{2}{*}{$\begin{array}{l}\text { RSG-895 } \\
\text { RSG-888 }\end{array}$} & $\mathrm{x}_{\mathrm{IRG}}$ & $0.30 \pm 2.23$ & $5.50 * \pm 2.35$ & $6.02 *$ & $17.00 \pm 0.47$ & $0.79 * \pm 0.35$ & $-3.07 \pm 1.68$ & $-2.04 \pm 1.67$ & $6.93 \pm 4.82$ & \\
\hline & $\mathrm{RF}$ & $-0.24 \pm 2.42$ & $5.96^{* *} \pm 2.17$ & $7.71^{*}$ & $16.60 \pm 0.46$ & $0.26 \pm 0.44$ & $-2.73 \pm 1.55$ & $-3.49 * \pm 1.60$ & $8.27 \pm 4.67$ & \\
\hline $\begin{array}{l}\text { RSG-888 } \\
\text { ICC-4958 }\end{array}$ & \multicolumn{2}{|c|}{$\mathrm{x}_{\text {Pooled } 4.67 * * \pm 1.67}$} & $9.56^{* *} \pm 1.83$ & $42.89^{* *}$ & $24.70 \pm 0.36$ & $-6.09 * * \pm 0.32$ & $-2.27 \pm 1.28$ & \multicolumn{2}{|c|}{$-17.78^{* *} \pm 1.276 .52 \pm 3.62$} & \\
\hline $\begin{array}{l}\text { IPC-94-94 } \\
\text { RSG-888 }\end{array}$ & \multicolumn{2}{|c|}{$\mathrm{x}_{\text {Pooled } 3.28 \pm 1.81}$} & $4.90 * * \pm 1.44$ & $19.61 * *$ & $22.38 \pm 0.36$ & $4.35^{* *} \pm 0.28$ & $-0.02 \pm 1.08$ & $5.97 * * \pm 1.19$ & $2.16 \pm 3.46$ & \\
\hline \multirow[t]{2}{*}{$\begin{array}{l}\text { CSJD-901 } \\
\text { RSG-931 }\end{array}$} & $\mathrm{x}_{\mathrm{IRG}}$ & $1.35 \pm 1.66$ & $4.72^{* *} \pm 1.43$ & $14.78 * *$ & $17.14 \pm 0.37$ & $0.69 * \pm 0.28$ & $-1.52 \pm 1.05$ & $-1.54 \pm 1.17$ & $4.49 \pm 3.36$ & - \\
\hline & $\mathrm{RF}$ & $2.19 \pm 1.88$ & $6.39^{* *} \pm 2.25$ & $9.10^{*}$ & $16.48 \pm 0.27$ & $-0.80 \pm 0.45$ & $-2.40 \pm 1.50$ & $-5.50 * * \pm 1.49$ & $5.60 \pm 3.81$ & - \\
\hline BG-362 x RSG-931 & \multicolumn{2}{|c|}{1 Pooled $7.63 * * \pm 1.36$} & $7.79^{* *} \pm 0.19$ & $79.72 * *$ & $23.89 \pm 0.25$ & $3.78 * * \pm 0.32$ & $0.95 \pm 0.80$ & $3.65 * * \pm 0.97$ & $0.21 \pm 2.48$ & - \\
\hline \multicolumn{11}{|l|}{ Protein content (\%) } \\
\hline $\begin{array}{l}\text { RSG-895 } \\
\text { RSG-888 }\end{array}$ & \multicolumn{2}{|c|}{$\mathrm{x}_{\text {Pooled }-1.04 * \pm 0.51}$} & $-1.93^{* *} \pm 0.50$ & $21.74 * *$ & $17.64 \pm 0.10$ & $0.71^{* *} \pm 0.10$ & $1.08 * * \pm 0.35$ & $2.53 * * \pm 0.36$ & $-1.19 \pm 1.01$ & - \\
\hline $\begin{array}{l}\text { RSG- } 888 \\
\text { ICC-4958 }\end{array}$ & \multicolumn{2}{|c|}{$\mathrm{x}_{\text {Pooled- }} 0.52 \pm 0.51$} & $1.30 * * \pm 0.47$ & $8.01^{*}$ & $18.48 \pm 0.10$ & $-0.71 * \pm 0.10$ & $0.28 \pm 0.33$ & $-2.38^{* *} \pm 0.36$ & $2.44 * \pm 0.99$ & - \\
\hline \multirow[t]{2}{*}{$\begin{array}{l}\text { IPC-94-94 } \\
\text { RSG-888 }\end{array}$} & $\mathrm{x}_{\mathrm{IRG}}$ & $-2.25 * * \pm 0.52$ & $-4.63 * * \pm 0.49$ & $120.19 * *$ & $18.25 \pm 0.10$ & $1.57 * * \pm 0.10$ & $2.21 * * \pm 0.34$ & $5.84 * * \pm 0.36$ & $-3.17 * * \pm 1.01$ & ( \\
\hline & $\mathrm{RF}$ & $-2.18 * * \pm 0.53$ & $-1.10 * \pm 0.48$ & $25.17 * *$ & $18.38 \pm 0.10$ & $1.50^{* *} \pm 0.10$ & $-0.16 \pm 0.34$ & $3.37 * * \pm 0.37$ & $1.44 \pm 1.03$ & - \\
\hline \multirow[t]{2}{*}{$\begin{array}{l}\text { CSJD-901 } \\
\text { RSG-931 }\end{array}$} & $\mathrm{x}_{\mathrm{IRG}}$ & $-1.62 * \pm 0.64$ & $0.64 \pm 0.51$ & $6.60^{*}$ & $18.19 \pm 0.13$ & $1.25^{* *} \pm 0.10$ & $-1.01 * * \pm 0.38$ & $1.80 * * \pm 0.43$ & $3.01 * \pm 1.25$ & 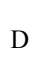 \\
\hline & $\mathrm{RF}$ & $-1.46^{* *} \pm 0.51$ & $-1.00 * \pm 0.47$ & $15.05 * *$ & $18.60 \pm 0.10$ & $0.32 * * \pm 0.09$ & $-0.65 \pm 0.33$ & $1.06 * * \pm 0.35$ & $0.61 \pm 0.99$ & - \\
\hline \multirow[t]{2}{*}{ BG-362 x RSG-931 } & IRG & $-0.88 \pm 0.53$ & $-1.94 * * \pm 0.57$ & $16.24 * *$ & $18.63 \pm 0.10$ & $0.97 * * \pm 0.10$ & $1.41 * * \pm 0.40$ & $3.09 * * \pm 0.40$ & $-1.41 \pm 1.11$ & \\
\hline & RF & $0.07 \pm 0.51$ & $-1.37 * * \pm 0.47$ & $8.65^{* *}$ & $19.25 \pm 0.10$ & $0.20 * \pm 0.10$ & $0.80 * \pm 0.33$ & $1.33 * * \pm 0.36$ & $-1.92 \pm 0.99$ & \\
\hline
\end{tabular}

$*$, ** significant at 5 per cent and 1 per cent level, respectively 


\subsection{Plant Height}

Main effects, additive (d) and dominance (h) were significant in all the crosses under both the conditions except CSJD-901x RSG-931 under rainfed condition, with greater magnitude of dominance than additive component, indicating predominance of dominance effect for this trait. Both additive $\mathrm{x}$ additive (i) and dominance $\mathrm{x}$ dominance (l) effects were equally important in the inheritance of this trait. However, the magnitude of dominance $\mathrm{x}$ dominance effect prevailed over the additive $\mathrm{x}$ additive effect in all the crosses under both the conditions except RSG-888 x ICC-4958 and IPC-94-94 x RSG-888 under irrigated condition, indicating preponderance of dominance $\mathrm{x}$ dominance effect in the inheritance of this trait.

\subsection{Fruiting Branches per Plant}

Both additive (d) and dominance (h) effects were significant in all the crosses under both the conditions except for additive effect in BG-362 x RSG-931 under rainfed condition. The higher magnitude of dominance than additive effect in most of the crosses indicate importance of dominance effect in the inheritance of this trait. Additive $\mathrm{x}$ additive (i) and dominance $\mathrm{x}$ dominance (1) interactions were also significant in all the crosses under both the conditions except additive $x$ additive (i) in RSG-895 x RSG-888 under irrigated, CSJD-901 x RSG-931 under both the conditions and dominance $\mathrm{x}$ dominance (1) in CSJD-901 x RSG-931 under irrigated condition. Higher magnitude of dominance $\mathrm{x}$ dominance (1) than additive $\mathrm{x}$ additive (i) effect, indicated the importance of dominance $\mathrm{x}$ dominance effect.

\subsection{Pods per Plant}

The main effects, additive (d) and dominance (h) were important in all the crosses except for dominance in IPC-94-94 x RSG-888 under rainfed condition. However, relative magnitude and desirable positive signs revealed the preponderance of dominance (h) over the additive (d) effect, indicating importance of dominance (h) effect for this trait under both the conditions. Among the interaction effects, additive $\mathrm{x}$ additive (i) and dominance $\mathrm{x}$ dominance (l) interactions were significant for all the crosses under both the conditions except for additive $\mathrm{x}$ additive (i) in RSG-895 x RSG-888 and CSJD-901 x RSG-931 under rainfed condition. Higher magnitude of dominance $\mathrm{x}$ dominance (l) than additive $\mathrm{x}$ additive (i) interaction indicated that dominance $\mathrm{x}$ dominance (l) interaction was important for this trait under both the conditions.

\subsection{Seeds per Pod}

Dominance gene effects were significant for seeds per pod in all the crosses in both the conditions as well as in pooled analysis over environments except IPC-94-94 x RSG-888 under both the conditions and additive effects were significant only in RSG-888 x ICC-4958 under pooled, IPC-94-94 x RSG-888 under rainfed and in CSJD-901 x RSG-931 under irrigated condition, indicating importance of dominance effect in the inheritance of this trait. Among the interaction effects, additive $x$ additive (i) gene effect was significant in all the crosses under both irrigated and rainfed conditions as well as in pooled analysis over environments, whereas dominance $\mathrm{x}$ dominance (1) gene action was significant only in two crosses i.e., RSG-895 x RSG-888 and RSG-888 x ICC-4958 under pooled analysis over environments, indicating the importance of additive $x$ additive (i) effect in the inheritance of this trait.

\subsection{Biological Yield per Plant}

WIth regards to main effects both the main effects i.e., additive (d) and dominance (h) were important for the inheritance of this trait in all the crosses under both the conditions except for dominance in IPC-94-94 $x$ RSG-888 under rainfed and in CSJD-901 x RSG-931 under irrigated condition. However, the magnitude of dominance (h) effect was higher than additive effect-indicating predominance of dominance effect for this trait. Similar, reports were also reported by Bhardwaj and Sandhu (2007) and Gupta et al. (2007). Additive x additive (i) and dominance $\mathrm{x}$ dominance (l) effects were also important for the inheritance of this trait in all the crosses under both the conditions except for additive $\mathrm{x}$ additive (i) in RSG-888 x ICC-4958 and dominance $\mathrm{x}$ dominance (1) in BG-362 x RSG-931 under irrigated condition. The magnitude of dominance $\mathrm{x}$ dominance (1) was higher than the additive $\mathrm{x}$ additive (i) which- indicated that dominance $\mathrm{x}$ dominance interaction played greater role in controlling of this trait.

\subsection{Seed Yield per Plant}

Both main effects i.e., additive (d) and dominance (h) were significant in all the crosses under both the conditions except for dominance in RSG-895 x RSG-888 and RSG-888 x ICC-4958 under irrigated condition. The magnitudes of dominance (h) were higher than their respective additive effect, indicating predominance of dominance effect for the inheritance of seed yield per plant. Additive $\mathrm{x}$ additive (i) and dominance $\mathrm{x}$ dominance (l) effects were also significant in all crosses under both the conditions except for additive $\mathrm{x}$ additive (i) in 
IPC-94-94 x RSG-888 under irrigated and CSJD-901 x RSG-931 under both the conditions with a greater magnitude of dominance $\mathrm{x}$ dominance interaction which- indicated the importance of dominance $\mathrm{x}$ dominance (l) interaction in controlling the inheritance of this trait.

\subsection{Harvest Index}

Both the main effects additive (d) and dominance (h) were significant in all the crosses under both the conditions except for dominance (h) in RSG-888 x ICC-4958 under rainfed, additive (d) in CSJD-901 x RSG-931 under irrigated and both additive (d) and dominance (h) in BG-362 x RSG-931 under both the conditions with a greater magnitude of dominance than additive effect- indicating predominance of dominance effect for the inheritance of this trait in both rainfed and irrigated conditions. The digenic interactions (i) and (1) were significant in all the cases except for additive $x$ additive (i) in RSG-895 x RSG-888 under rainfed and CSJD-901 x RSG-931 under irrigated and for dominance $x$ dominance (1) in RSG-888 x ICC-4958 and BG-362 x RSG-931 under irrigated condition with a greater magnitude of dominance $\mathrm{x}$ dominance (1), indicating importance of dominance $\mathrm{x}$ dominance (1) for the inheritance of this trait.

\subsection{0-seed Weight}

Among the main effects, additive effect (d) was significant in all the crosses in both the conditions as well as in pooled analysis over environments (irrigated and rainfed) except RSG-895 x RSG-888 and CSJD-901 x RSG931 under rainfed, whereas dominance $(\mathrm{h})$ effect was non-significant in all the cases- indicating importance of additive effect in the inheritance of 100-seed weight. Among the interaction effects, additive $\mathrm{x}$ additive (i) effect was significant in all the crosses under both the conditions as well as in pooled analysis over environments, except in RSG-895 x RSG-888 and CSJD-901 x RSG-931 under irrigated, whereas dominance $x$ dominance (1) effect was found non-significant in all the cases- indicating role of additive $\mathrm{x}$ additive (i) in controlling the inheritance of this trait.

\subsection{Protein Content}

The main effects additive (d) and dominance (h) were important for this trait, since both were significant in all the cases except for dominance (h) in RSG-888 x ICC-4958 in pooled analysis over environments and in IPC-94-94 x RSG-888 and CSJD-901 x RSG-931 under rainfed. Higher magnitude of dominance than additive effect in most of the cases indicated the greater role of dominance effect in the inheritance of this trait. Additive $\mathrm{x}$ additive (i) was significant in all the eight cases, however, dominance $\mathrm{x}$ dominance (l) was significant in only three cases under both the conditions including pooled analysis over environments-which indicated importance of additive $\mathrm{x}$ additive interaction for the inheritance of this trait.

In all the crosses under both the conditions, non-allelic interactions were predominant for most of the characters. The non-additive gene effects played greater role in the inheritance of all the characters under both the conditions except for 100-seed weight. Earlier Singh and Ramanujam (1981), Patil et al. (1987), Pandey and Tiwari (1989), Shinde and Deshmukh (1990), Mandal (1992), Girase and Deshmukh (2000), Mehla et al. (2000), Singh et al. (2001), Patil et al. (2004), Bhardwaj and Sandhu (2007), Gupta et al. (2006) and Gupta et al. (2007) also reported that both additive and non-additive gene actions for yield and yield contributing characters in chickpea.

\section{Conclusion}

In the present study non-additive gene effects viz., dominance (h) and dominance $\mathrm{x}$ dominance (l) was controlling the expression of most of the characters under both the conditions and it is suggested that breeders should follow such methods which can mop-up the genes to form superior gene constellations interacting in a favorable manner. Hence, it is suggested that recurrent selection by way of intermating the desirable segregants followed by selection or biparental approach/intermating of segregants in early segregating generations for improvement of yield contributing characters should be followed.

\section{References}

Bhardwaj, R., \& Sandhu, J. S. (2007). Gene effects for yield and its components in chickpea. Crop Improvement, $34(1), 48-51$.

Cavalli, L. L. (1952). An analysis of linkage in quantitative inheritance. In E. C. R. Reeve, \& C. H. Waddington (Eds.) (pp. 135-144), Quantitative Inheritance HMSC. London.

Deshmukh, R. B., \& Bhapkar, D. G. (1982). Heterosis and combining ability for yield and its components in chickpea. Indian Journal of Agricultural Sciences, 52(11), 728-731. 
Girase, V. S., \& Deshmukh, R. B. (2000). Gene action for yield and its components in chickpea. Indian Journal of Genetics \& Plant Breeding, 60(2), 185-189.

Gupta, S. K., Ajinder K., \& Sandhu, J. S. (2007). Combining ability for yield and its components in Kabuli chickpea. Crop Improvement, 34(1), 52-55.

Gupta, S. K., Sandhu, J. S., \& Garg, T. (2006). Combining ability in desi chickpea. Indian Journal of Pulses Research, 20(1), 22-24.

Hayman, B. I. (1958). The separation of epistatic from additive and dominance variation in generation means. Heredity, 12, 371-390. http://dx.doi.org/10.1038/hdy.1958.36

Katiyar, R. P., Singh, D., \& Verma, V. S. (1980). Heterosis response and inbreeding depression in chickpea. Tropical Grain Legumes Bulletin, 19, 41-43.

Mandal, A. K. (1992). Pattern of variation of harvest index in chickpea crosses. Indian Journal of Genetics and Plant Breeding, 52(2), 164-168.

Mather, K. (1949). Biometrical Genetics (p. 162). Ist Ed., London: Mathuen and Company, Ltd.

Mehla, I. S., Singh, V. P., Waldia, R. S., Surat, S., \& Singh, S. (2000). Inheritance of grain yield and its components in Kabuli gram (Cicer arietinum L.). Legume Research, 23(3), 163-166.

Pandey, R. L., \& Tiwari, A. S. (1989). Estimation of gene effects and heterosis in chickpea. Indian Journal of Agricultural Research, 23(4), 191-199.

Panse, V. G., \& Sukhatme, P. V. (1985). Statistical Methods for Agricultural Workers (2nd Ed.). Indian Council of Agricultural Research, Publication, New Delhi.

Patil, J. V., Kulkarni, S. S., \& Gawande, V. L. (2004). Genetics of quantitative characters in chickpea (Cicer arietinum L.). National Journal of Plant Improvement, 6(2), 96-99.

Patil, V. D., Nerkar, Y. S., \& Dayama, D. K. (1987). Inheritance of quantitative characters in chickpea. Indian Journal of Agricultural Sciences, 57(2), 78-81.

Shinde, N. V., \& Deshmukh, R. B. (1990). Inheritance of quantitative characters in chickpea. Indian Journal of Genetics and Plant Breeding, 50(4), 342-347.

Singh, S. P., \& Ramanujam, S. (1981). Gene action and heterosis in Bengal gram. Indian Journal of Genetics and Plant Breeding, 41(1), 150-153.

Singh, Shoraj., Kumar, J., Yadav, S. S., \& Singh, S. (2001). Genetic analysis of agronomic traits in chickpea. New-Botanist, 28(1-4), 5-11.

Toledo, J., De, F. F., Rossini, M. C., Souza, R., De, F., \& Leao, F. F. (1991). Genetical analysis of soybean biparental cross and comparative model fitting to means and variances of two sets of germination. Revista Brasileria de Genetica, 14, 1041-1064. 